\title{
Oral Anticoagulation Therapy in Heart Failure Patients in Sinus Rhythm: A Systematic Review and Meta-Analysis
}

\author{
Giuseppe Rengo ${ }^{1,2 * 9}$, Gennaro Pagano ${ }^{2 * 9}$, Alessandro Squizzato ${ }^{3}$, Lorenzo Moja ${ }^{4,5}$, Grazia \\ Daniela Femminella ${ }^{2}$, Claudio de Lucia ${ }^{2}$, Klara Komici ${ }^{2}$, Valentina Parisi ${ }^{2}$, Gianluigi Savarese ${ }^{6}$, \\ Nicola Ferrara ${ }^{1,2}$, Pasquale Perrone-Filardi ${ }^{6}$, Dario Leosco ${ }^{2}$
}

1 Division of Cardiology, "Salvatore Maugeri" Foundation, IRCCS - Scientific Institute of Telese Terme, Benevento, Italy, 2 Department of Medical Translational Sciences, Federico II University of Naples, Naples, Italy, $\mathbf{3}$ Research Center on Thromboembolic Disorders and Antithrombotic Therapies, Department of Clinical and Experimental Medicine, University of Insubria, Varese, Italy, 4 Department of Biomedical Sciences for Health, University of Milan, Milan, Italy, 5 IRCCS Galeazzi Orthopedic Institute, Milan, Italy, $\mathbf{6}$ Department of Advanced Biomedical Sciences, Federico II University of Naples, Naples, Italy

\begin{abstract}
Background: Heart failure (HF) patients show high morbidity and mortality rate with increased risk of malignant arrhythmia and thromboembolism. Anticoagulation reduces embolic event and death rates in HF patients with atrial fibrillation, but if antithrombotic therapy is beneficial in patients with HF in sinus rhythm is still debated.

Methodology and Principal Findings: We conducted a systematic review of prospective, randomized controlled trials (RCTs) to assess the efficacy and safety of oral anticoagulant therapies (OATs) compared to antiplatelet treatment in HF patients in sinus rhythm. MEDLINE, Web of Science, CENTRAL and Scopus databases were searched up to May 2012. Four RCTs were identified and a total of 3663 patients were included in the meta-analysis. Patients with both ischemic and nonischemic HF were included. There was no significant difference in mortality (odds ratio (OR) $1.01,95 \%$ confidence interval (CI) 0.86 to 1.19) between OATs group and antiplatelet drug group. OATs have reduced ischemic stroke risk $(\mathrm{OR} 0.49,95 \% \mathrm{Cl}$ 0.32 to 0.74 ), but have increased major bleeding risk (OR $2.01,95 \% \mathrm{Cl} 1.40$ to 2.88 ) compared to antiplatelet treatment.

Conclusion: In HF patients in sinus rhythm OATs do not show a better risk-benefit profile compared to antiplatelet treatment in cardioembolism prevention. Warfarin and aspirin seem to be similar in reducing mortality. Warfarin reduces the incidence of ischemic stroke, but increases major bleedings. Thus, it is possible to speculate that aspirin prescription be indicated in patients with high risk of bleeding, whereas warfarin could be preferred in patients with high thromboembolic risk.
\end{abstract}

Citation: Rengo G, Pagano G, Squizzato A, Moja L, Femminella GD, et al. (2013) Oral Anticoagulation Therapy in Heart Failure Patients in Sinus Rhythm: A Systematic Review and Meta-Analysis. PLoS ONE 8(1): e52952. doi:10.1371/journal.pone.0052952

Editor: Adrian V. Hernandez, Universidad Peruana de Ciencias Aplicadas (UPC), Peru

Received September 21, 2012; Accepted November 22, 2012; Published January 2, 2013

Copyright: (C) 2013 Rengo et al. This is an open-access article distributed under the terms of the Creative Commons Attribution License, which permits unrestricted use, distribution, and reproduction in any medium, provided the original author and source are credited.

Funding: The authors have no support or funding to report.

Competing Interests: The authors have declared that no competing interests exist.

* E-mail: giuseppe.rengo@unina.it (GR); gennaropagano85@hotmail.com (GP)

9 These authors contributed equally to this work.

\section{Introduction}

Heart failure (HF) is a growing public health problem worldwide, and it is associated with an increased risk of left ventricular thrombus formation and cerebral embolism due to endothelial dysfunction, reduced blood flow and underlying state of hypercoagulability [1-4]. In the population-based Framingham Heart Study, the relative risk of stroke in individuals with HF compared to those without $\mathrm{HF}$ was 4.1 for men and 2.8 for women [5]. The risk of cardioembolism is further enhanced by the presence of atrial fibrillation (AF), however $\mathrm{HF}$ patients in sinus rhythm still have higher thromboembolic risk. A retrospective analyses reports a yearly incidence of thromboembolism of $1.0 \%-4.5 \%$ in HF patients without AF [6]. In the SAVE study, an observational analysis of 2231 patients with left ventricular dysfunction after acute myocardial infarction, $4.6 \%$ of patients had fatal or non fatal strokes during the study period (rate of stroke per year of follow-up, 1.5 percent) and the estimated five-year stroke rate was 8.1 percent in the entire population $[7,8]$. Antiplatelet therapy is commonly prescribed in HF patients in sinus rhythm since ischemic cardiomyopathy is the principal underlying cause [9,10]. Conversely, oral anticoagulant therapy (OAT), that includes oral vitamin $\mathrm{K}$ antagonists (VKAs) and new oral anticoagulant therapies, is commonly prescribed in $\mathrm{HF}$ patients with $\mathrm{AF}$ since it has been shown more efficacious than aspirin in reducing embolic risk $[9,11]$. International guidelines recommend the use of VKAs in HF patients with $\mathrm{AF}$ to prevent cardioembolic risk but $\mathrm{OAT}$ is not indicated in $\mathrm{HF}$ patients without $\mathrm{AF}[12,13,14]$.

The aim of the present meta-analysis has been to assess the efficacy and safety of OAT in comparison to antiplatelet treatment in $\mathrm{HF}$ patients in sinus rhythm.

\section{Methods}

The study was designed according to the PRISMA (Preferred Reporting Items for Systematic Reviews and Meta-Analyses) statement [15]. 


\section{Search Strategy}

MEDLINE, Web of Science, Cochrane CENTRAL, Scopus databases were searched for articles in all languages published until May 2012. Gray literature was not considered as a priority asset of our systematic review. Studies were identified and evaluated by the authors (GR, GP, AS) using the major medical subject heading combined with text and key words. As example for MEDLINE ("heart failure" [MeSH Terms] OR "heart failure"[All Fields] OR ("heart"[All Fields] AND "failure"[All Fields])) AND (“anticoagulants" [MeSH Terms] OR "anticoagulants"[All Fields] OR ("anti"'[All Fields] AND "coagulant"[All Fields]) OR "anti coagulant”[All Fields] OR "warfarin" [MeSH Terms] OR “warfarin"[All Fields] OR "antithrombins" [MeSH Terms] OR "antithrombins"[All Fields] OR "antithrombin"[All Fields] OR "aspirin" [MeSH Terms] OR "aspirin"[All Fields]). Additional eligible studies were identified screening the reference lists of studies included in our analysis.

\section{Study Selection}

All selected titles and abstracts were independently reviewed by two authors (GP,GR). Studies were excluded if the title and/or abstract were not appropriate for the aim of our review. Full texts were subsequently obtained for eligible studies or when the relevance of an article could not be certainty excluded. Disagreement was resolved by consensus and by opinion of a third reviewer (AS), when necessary. Selected studies were eligible if they met the following criteria: patients with heart failure due to any underlying cause without AF; adults only; patients treated with OAT or antiplatelet treatment; at least 100 patients enrolled; duration of treatment at least 1 month; RCT design. Reviews, case-reports, non-human studies and abstracts or conference proceedings were excluded. In summary, the present meta-analysis included only RCTs that compared the efficacy and the safety of OAT versus antiplatelet treatment among HF patients in sinus rhythm.

\section{Risk of Bias in Included Studies}

Using the guidelines in the Cochrane Handbook for assessment of risk of bias (RoB), RCTs were graded by two independent reviewers (GR and GP) basing on sequence generation, allocation concealment, incomplete outcome data, selective outcome reporting, blinding of participants and personnel and blinding of outcome assessment [16]. These items were considered as key domains for RoB assessment and classified as "adequate" (low risk of bias), "inadequate" (high risk of bias), or "unclear". Studies with adequate procedures in all domains were considered to have a low risk of bias; ones with inadequate procedures in one or more key domain(s) were considered to have a high risk of bias; and ones with unclear procedures in one or more key domain $(\mathrm{s})$ were considered to have an unclear risk of bias. Disagreement was resolved by consensus and by opinion of a third reviewer (AS).

\section{Data Extraction and Types of Outcomes Measures}

Data extraction has been completed by two reviewers (GR, GP) independently using a standardized form. Disagreement was resolved by consensus and by the opinion of a third reviewer (LM), when necessary. Overall mortality was the primary outcome. Additional efficacy outcomes were: ischemic stroke, myocardial infarction (MI), hospital admission. Main safety outcomes were: major bleedings and intracerebral bleedings. A separated analysis was planned for HF patients with ischemic cardiomyopathy and with non-ischemic cardiomyopathy. To define the severity of bleeding events we planned to use the
International Society of Thrombosis and Haemostasis (ISTH) classification [17].

\section{Statistical Analysis}

Odds ratios (OR) and 95\% confidence intervals (CI) were calculated. The results were pooled using the inverse variance method. The random effect model described by DerSimonian and Laird [18] was used to synthesize data rather than the fixed effect model because it incorporates intra- and inter-study variability. The software Review Manager (RevMan, version 5.1.4 for Windows 7, Copenhagen: The Nordic Cochrane Centre, The Cochrane Collaboration, 2011) supported the analysis.

\section{Assessment of Heterogeneity and Publication Bias}

Heterogeneity was assessed using $\mathrm{I}^{2}$ statistic that accounts of between-study (or inter-study) variability as opposed to withinstudy (or intra-study) variability. Because of latent clinical heterogeneity, random-effects model was used, independently of statistical evidence for heterogeneity. Heterogeneity has been considered substantial if $\mathrm{I}^{2}$ value was greater than $25 \%$ [19]. If substantial heterogeneity was identified, subgroup analyses and sensitivity analyses were performed. Presence of publication bias was explored performing the test for asymmetry of the funnel plot by Egger that is a linear regression of normalized effect estimate (estimate divided by its standard error) against precision (reciprocal of the standard error of the estimate) [20,21].

\section{Results}

Of 17625 articles identified by the initial search, 16 were retrieved for more detailed evaluation and 4 were finally included in the meta-analysis (Figure 1) [22-25]. All included studies have enrolled patients without atrial fibrillation However, we found three studies not reporting separated data for patients in sinus rhythm and in atrial fibrillation, thus they were excluded from meta-analysis [26-28]. Baseline characteristics of patients included in the studies were summarized in Table 1 . Studies population sizes ranged from 180 [22] to 2305 [25] patients, for a total of 3663 included patients. Quality assessment items have been summarized in Figure 2. The WASH study [22], the pilot study of WATCH study, included 279 patients that were randomized to warfarin (target INR 2.5), aspirin (325 mg), or placebo and followed up for a mean of 27 months. The HELAS trial [23] separated 197 patients according to the etiology of their HF. Only patients in the ischemic cardiomyopathy group (n.115) were randomized to receive warfarin (target INR 2-3) or aspirin $(325 \mathrm{mg})$, while patients in the non-ischemic group (n.82) were randomized to receive warfarin or placebo. Patients were followed up for a mean period of 20 months. We included in our metaanalysis only the ischemic group since it compared warfarin versus aspirin. The WATCH trial [24] included $1587 \mathrm{HF}$ patients receiving aspirin (162 mg), clopidogrel $(75 \mathrm{mg})$, or warfarin (target INR 2-3.5), followed up for a mean duration of 21 months. The WARCEF trial [25] randomized $2305 \mathrm{HF}$ patients to receive aspirin $(325 \mathrm{mg}$ ) or warfarin (target INR 2.5-3.5), with a mean follow up of 42 months.

\section{Efficacy Outcomes}

In the cumulative analysis of all patients $(n=3663)$, mortality rate was not significantly different between warfarin and aspirin groups (OR 1.01, 95\% CI 0,86 to 1,$19 ; \mathrm{I}^{2}=0 \%$ ). Warfarin was associated with a significant reduction of ischemic stroke compared to aspirin (OR $0.49,95 \%$ CI 0.32 to $0.74 ; \mathrm{I}^{2}=0 \%$, NNT $=50)$. Hospital admission and MI rates were not significantly 

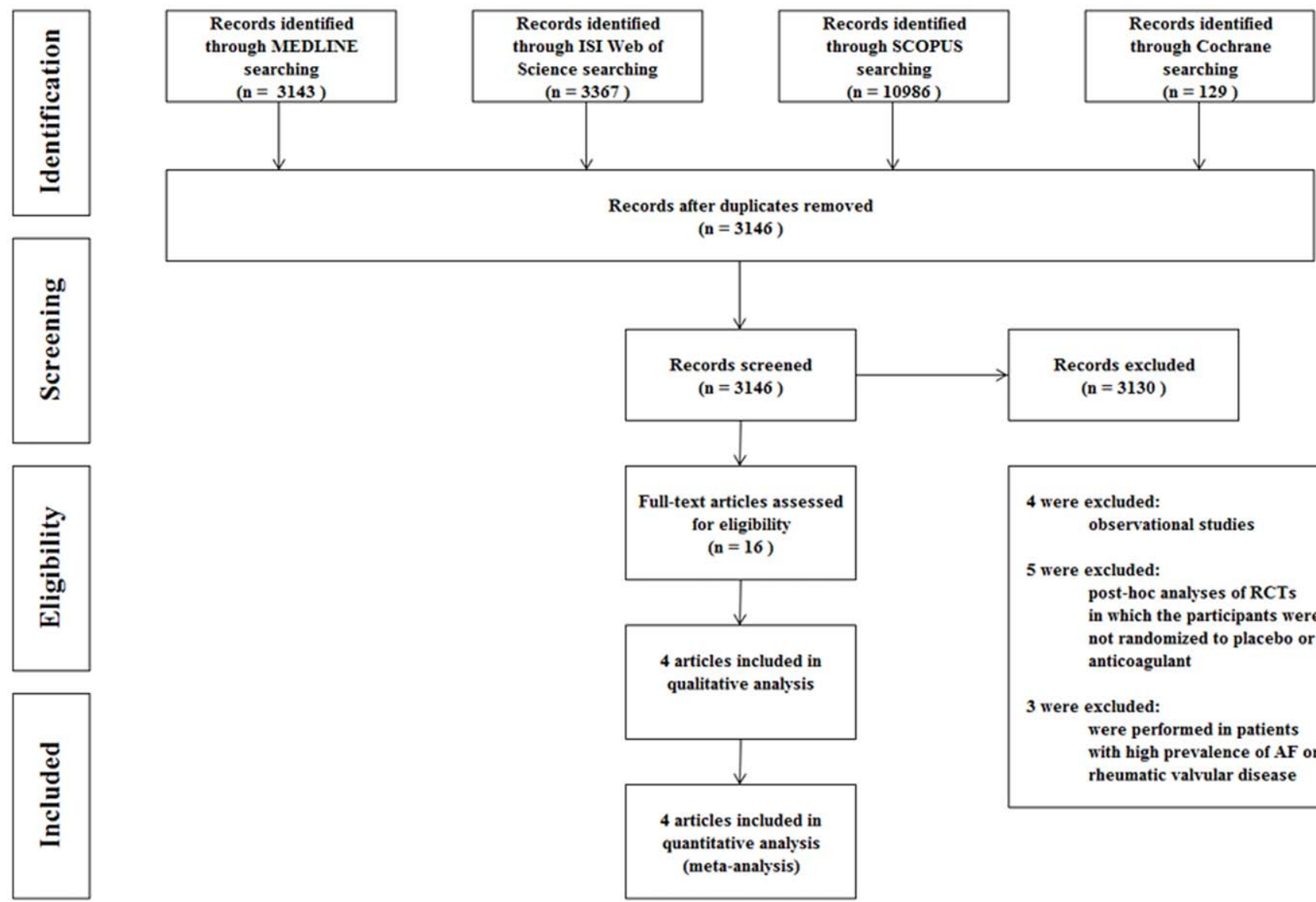

$(\mathrm{n}=3146)$

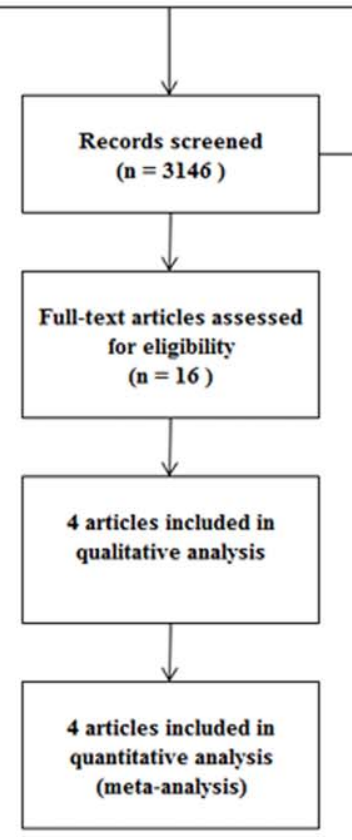

Records excluded

$(\mathrm{n}=3130)$

4 were excluded:

observational studies

5 were excluded:

post-hoc analyses of RCTs

in which the participants were

not randomized to placebo or

anticoagulant

3 were excluded:

were performed in patients with high prevalence of $A F$ or rheumatic valvular disease
Figure 1. Meta-analysis flow chart. doi:10.1371/journal.pone.0052952.g001

different between warfarin and aspirin groups (OR 0.80, 95\% CI 0.49 to $1.30, \mathrm{I}^{2}=80 \%$ and $\mathrm{OR} 1.00,95 \%$ CI 0.59 to 1.68 ; $\mathrm{I}^{2}=25 \%$ respectively) (Figure 3 ). One study removed analysis showed that, only when the WARCEF study was excluded from analysis, there was a reduced hospital admission rate in OAT compared to aspirin group with a statistically significant reduction in heterogeneity $\left(\mathrm{OR}=0.65\right.$, C.I. 0.50 to $0.85 ; \mathrm{I}^{2}=0 \%$, NNT = 15). Subgroup analyses in ischemic and non ischemic patients were not performed since only one study provided separated data for HF etiology [23].

\section{Safety Outcomes}

Data about bleeding events were not reported in enough trials for applying ISTH classification and the definition of major bleeding provided by authors was used. In the overall cohort of patients $(\mathrm{n}=3663)$, OAT was associated with significant increase in major bleeding events compared to aspirin (OR 2.01, 95\% CI 1.39 to $\left.2.89 ; \mathrm{I}^{2}=4 \%, \mathrm{NNH}=35\right)$. Moreover, a trend to increase in intracerebral bleedings has been observed in the warfarin group, but it was not statistically significant (OR 2.18, 95\% CI 0.75 to $\left.6.30 ; \mathrm{I}^{2}=0 \%\right)$.

\section{Publication Bias}

No publication bias was detected by Egger's linear regression method for each single outcomes analysis although the limited number of studies could not rule out a possible publication bias (data not shown).

\section{Discussion}

In the medical community there is not general consensus in either recommending or advising against OAT in HF. Several evidence have recommended OAT in $\mathrm{HF}$ patients with $\mathrm{AF}$, as prevention for the high cardioembolic risk observed in this population. Differently, the majority of $\mathrm{HF}$ patients due to ischemic etiology takes aspirin for secondary prevention of coronary heart disease. Therefore, the aim of this present metaanalysis has been to assess potential differences in efficacy and safety between these two therapeutic modalities in HF patients in sinus rhythm. Our results indicate that warfarin does not show a better efficacy-safety profile compared to aspirin in preventing cardioembolism in $\mathrm{HF}$ patients without AF. However, when compared to aspirin, warfarin was associated with a significant reduction (OR 0.49) of ischemic stroke incidence with a relative risk reduction that is comparable to that reported in HF patients with AF treated with OAT [29]. Nevertheless, due to the low annual stroke rate observed in HF patients in sinus rhythm (between $0.8 \%$ and $3.2 \%$ per year), the advantages deriving from routine anticoagulation cannot overcome the increased risk of bleedings related to warfarin use [24,30-33]. Consistently, our data show that OAT was associated with a more than doubled bleeding risk, with a trend to increase also the risk of intracerebral bleedings. It is important to underline, that only in the HELAS study, HF patients were dived for ischemic or non ischemic cardiomyopathy, thus no conclusions can be drown about efficacy or safety in these different patient subpopulations. 


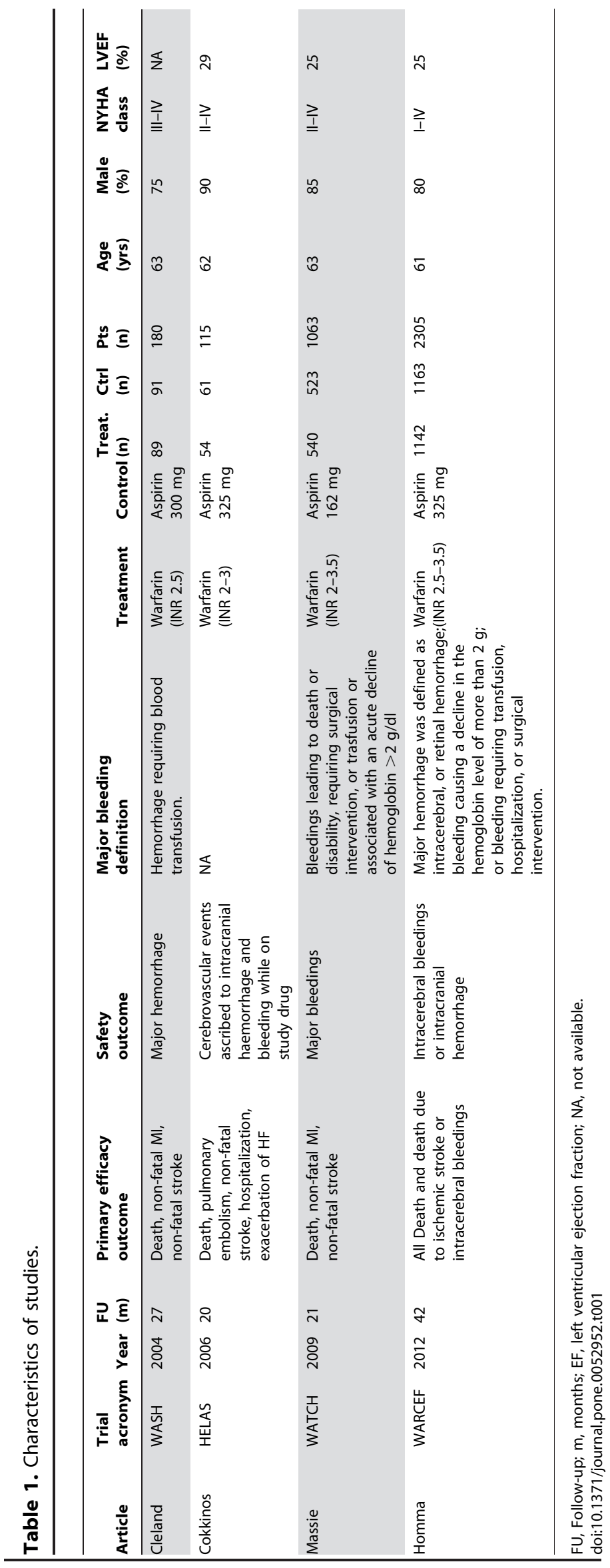




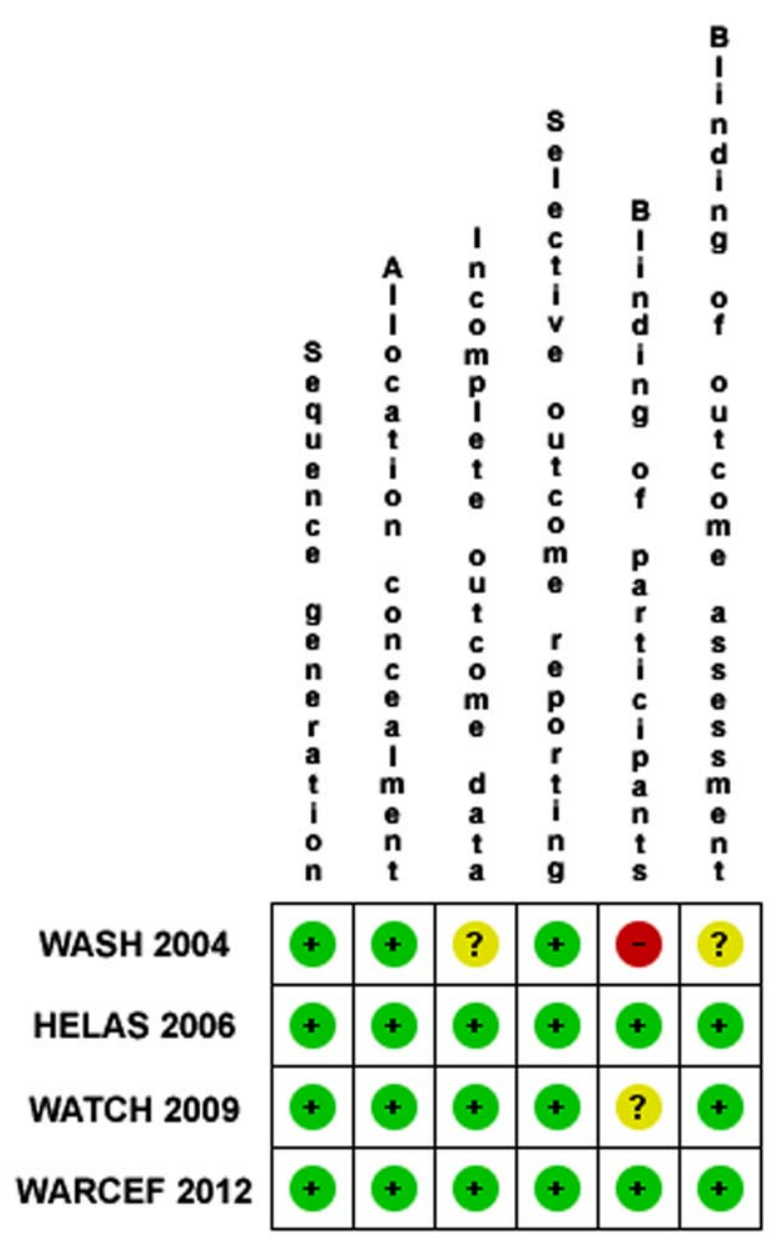

Figure 2. Risk of bias summary: review authors' judgment about each risk of bias item for each included study. doi:10.1371/journal.pone.0052952.g002

In $\mathrm{HF}$ patients with non ischemic etiology and without $\mathrm{AF}$, no studies are available about efficacy and safety of OAT or antiplatelet therapy compared to placebo, whereas several line of evidence support the use of aspirin in HF patients due to ischemic etiology as secondary prevention of coronary artery disease. Thus, RCTs comparing aspirin vs. placebo in HF patients in sinus rhythm and non ischemic etiology might be helpful to guide therapy in this specific HF subpopulation. In the present analysis the hospital admission rate for worsening $\mathrm{HF}$ was lower in warfarin group only after exclusion of WARCEF study. Its exclusion reduces the heterogeneity among studies but unfairly reduces the available information since this is the largest trial with the longest follow-up. The important issue regarding discordant results on the incidence of hospital admissions for worsening $\mathrm{HF}$ in patients treated with warfarin or aspirin is not addressable. Unfortunately, despite it would be of great interest whether warfarin treatment impacts quality of life (especially in terms of anxiety burden related to OAT monitoring), the identified trials did not investigate this relevant aspect which certainly would be an important argument for future trial investigations.

\section{Strengths and Limitations of this Meta-analysis}

The main strengths of our review include the systematic strategy and the high score at Cochrane quality assessment for all trials included. Our meta-analysis has one major shortcoming. It was not carried out on individual patients data exploring subgroups with higher thromboembolic risk. Moreover, there are some limitations in the outcome evaluation due to the too small sample size and the too short follow up (i.e. 2 years) period, resulting in a low number of events in the trials included. The exclusion of gray literature could be a limitation of our search strategy. It is important to underline that in WATCH trial, aspirin dosage is lower than that used in the other trials, thus explaining the lower efficacy of treatment reported. Although differences in trial definitions of outcomes among different trials should be considered for the interpretation of the overall result, it is important to mention that mortality and ischemic stroke, the principal efficacy outcomes evaluated in our study, are hard endpoint not affected by study definitions. The absence of heterogeneity in the most part of analysis supports the strength of our results. Further studies are needed to better identify high risk HF subgroups. The definition of an $\mathrm{HF}$ risk stratification score, similar to that available for ischemic risk assessment (CHADS2 or CHA2DS2-VASc scores), or bleeding risk assessment (HAS-BLED) [34,35], will be useful to identify $\mathrm{HF}$ patients at higher risk. Finally, no evidences are provided regarding the use of new oral anticoagulants (oral direct thrombin inhibitors, oral Factor Xa inhibitors) which seem to offer a different risk-benefit profile compared to warfarin and might induce a reduction in ischemic stroke rates with less risk of major bleeding. Thus, an head to head comparison between warfarin and new anticoagulants (rivaroxaban, apixaban and dabigatran),

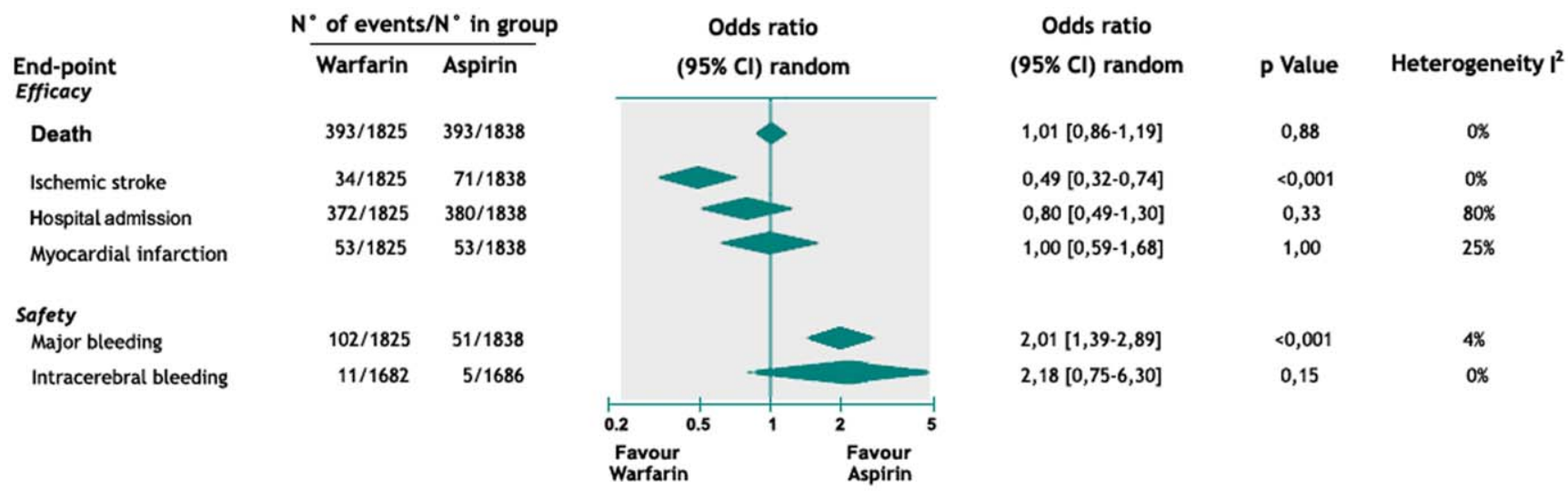

Figure 3. Pooled event rate and odds risk ratio for major end point in overall cohort patients with heart failure in sinus rhythm. doi:10.1371/journal.pone.0052952.g003 
with antiplatelet therapy might be of great interest in $\mathrm{HF}$ patients in sinus rhythm.

\section{Conclusions}

In patients with HF in sinus rhythm, warfarin and aspirin seem to be similar in reducing mortality. Warfarin reduces the incidence of ischemic stroke, but increases major bleedings. Thus, it is possible to speculate that aspirin could be indicated in patients with high risk of bleeding, whereas warfarin could be preferred in

\section{References}

1. Hunt SA, Abraham WT, Chin MH, Feldman AM, Francis GS, et al. (2009) 2009 Focused update incorporated into the ACG/AHA 2005 Guidelines for the Diagnosis and Management of Heart Failure in Adults. J Am Coll Cardiol; 53: e1-e90.

2. Roger VL, Go AS, Lloyd-Jones DM, Benjamin EJ, Berry JD, et al. (2012) Heart disease and stroke statistics - 2012 update: a report from the American Heart Association. Circulation;125(1): e2-e220.

3. Pilotto A, Addante F, Franceschi M, Leandro G, Rengo G, et al. (2010) Multidimensional Prognostic Index based on a comprehensive geriatric assessment predicts short-term mortality in older patients with heart failure. Circ Heart Fail;3(1): 14-20.

4. Lip GYH, Gibbs CR (1999) Does heart failure confer a hypercoagulable state? Virchow's triad revisited. J Am Coll Cardiol;33: 1424-6.

5. Kannel WB, Wolf PA, Verter J (1983) Manifestations of coronary disease predisposing to stroke. The Framingham study. JAMA;250: 2942-2946.

6. Isnard R, Komajda M (2001) Thromboembolism in heart failure, old ideas and new challenges. Eur J Heart Fail;3: 265-269.

7. Loh E, Sutton MS, Wun CC, Rouleau JL, Flaker GC, et al. (1997) Ventricular dysfunction and the risk of stroke after myocardial infarction. N Engl J Med;336: 251-7.

8. Freudenberger RS, Schumaecker MM, Homma S (2010) What is the appropriate approach to prevention of thromboembolism in heart failure? Thromb Haemost; 103: 489-95.

9. Antiplatelet Trialists' Collaboration (1994) Collaborative overview of randomised trials of antiplatelet therapy-I: Prevention of death, myocardial infarction, and stroke by prolonged antiplatelet therapy in various categories of patients. BMJ;308(6921): 81-106.

10. Antiplatelet Trialists' Collaboration (2002) Collaborative meta-analysis of randomised trials of antiplatelet therapy for prevention of death, myocardial infarction, and stroke in high risk patients. BMJ;324(7329): 71-86.

11. Agarwal S, Hachamovitch R, Menon V (2012) Current trial-associated outcomes with warfarin in prevention of stroke in patients with nonvalvular atrial fibrillation: a meta-analysis. Arch Intern Med;172(8): 623-31.

12. McMurray JJ, Adamopoulos S, Anker SD, Auricchio A, Böhm M, et al. (2012) ESC guidelines for the diagnosis and treatment of acute and chronic heart failure 2012: The Task Force for the Diagnosis and Treatment of Acute and Chronic Heart Failure 2012 of the European Society of Cardiology. Developed in collaboration with the Heart Failure Association (HFA) of the ESC. Eur J Heart Failure;14: 803-86.

13. Lip GY, Ponikowski P, Andreotti F, Anker SD, Filippatos G, et al. (2012) Thrombo-embolism and antithrombotic therapy for heart failure in sinus rhythm. A Joint Consensus Document from the ESC Heart Failure Association and the ESC Working Group on Thrombosis. Eur J Heart Fail;14: 681-95.

14. Hirsh J, Fuster V, Asell J, Halperin JL, American Heart Association/American College of Cardiology Foundation (2003) American Heart Association/ American College of Cardiology Foundation guide to warfarin therapy. J Am Coll Cardiol;41: 1633-52.

15. Moher D, Liberati A, Tetzlaff J, Altman DG, The PRISMA Group (2009) Preferred Reporting Items for Systematic Reviews and Meta-Analyses: The PRISMA Statement. PLoS Med 6(6): e1000097. doi:10.1371/journal.pmed 1000097 .

16. Higgins JPT, Green S (2011) Cochrane Handbook for Systematic Reviews of Interventions. Available: www.cochrane-handbook.org. Accessed 2011 Mar 20.

17. Schulman S, Kearon C (2005) On behalf of the subcommittee on control of anticoagulation of the Scientific and Standardization committee of the International Society on Thrombosis and Haemostasis. Definition of major bleeding in clinical investigations of antihemostatic medicinal products in nonsurgical patients. Scientific and Standardization Committee Communication. J Thromb Haemost;3: 692-4. patients with high thromboembolic risk. However, further studies are needed to clarify the role of antitrombotic therapy in $\mathrm{HF}$ patients in sinus rhythm, particularly in the subpopulation with non ischemic etiology.

\section{Author Contributions}

Conceived and designed the experiments: GP LM AS. Analyzed the data: GP GDF CdL KK NF PPF DL GS VP. Wrote the paper: GP GR AS LM.

18. DerSimonian R, Laird N (1986) Meta-analysis in clinical trials. Control Clin Trials; 7: 177-188.

19. Higgins JP, Thompson SG, Deeks JJ, Altman DG (2003) Measuring inconsistency in meta-analyses. BMJ;327: 557-560.

20. Sterne JA, Egger M, Smith GD (2001) Systematic reviews in health care: investigating and dealing with publication and other biases in meta-analysis. BMJ;323: 101-105.

21. Egger M, Smith GD, Phillips AN (1997) Meta-analysis: principles and procedures. BMJ;315: 1533-7.

22. Cleland JG, Findlay I, Jafri S, Sutton G, Falk R, et al. (2004) The warfarin/ aspirin study in heart failure (WASH): a randomized trial comparing antithrombotic strategies for patients with heart failure. Am Heart J;148: 157164.

23. Cokkinos DV, Haralabopoulos GC, Kostis JB, Toutozas PK, HELAS Investigators (2006) Efficacy of antithrombotic therapy in chronic heart failure: the HELAS study. Eur J Heart Fail;8: 428-432.

24. Massie BM, Collins JF, Ammon SE, Armstrong PW, Cleland JG, et al. (2009) Randomized trial of warfarin, aspirin, and clopidogrel in patients with chronic heart failure: the Warfarin and Antiplatelet Therapy in Chronic Heart Failure (WATCH) trial. Circulation;119: 1616-1624

25. Homma S, Thompson JL, Pullicino PM, Levin B, Freudenberger RS, et al (2012) Warfarin and Aspirin in Patients with Heart Failure and Sinus Rhythm. N Engl J Med doi:10.1056/NEJMoa1202299.

26. Anderson GM, Hull E (1950) The effects of dicumarol upon the mortality and incidence of thromboembolic complications in congestive heart failure. Am Heart J;39: 697-702.

27. Griffith GC, Stragnell R, Levinson DC, Moore FJ, Ware AG (1952) A study of the beneficial effects of anticoagulant therapy in congestive heart failure. Ann Intern Med;37: 867-87.

28. Harvey WP, Finch CA (1950) Dicumarol prophylaxis of thromboembolic disease in congestive heart failure. N Engl J Med;242: 208-11.

29. Hart RG, Benavente O, McBride R, Pearce LA (1999) Antithrombotic therapy to prevent stroke in patients with atrial fibrillation: a meta-analysis. Ann Intern Med;131: 492-501.

30. Gottdiener JS, McClelland RL, Marshall R, Shemanski L, Furberg CD, et al. (2002) Outcome of congestive heart failure in elderly persons: influence of left ventricular systolic function. The Cardiovascular Health Study. Ann Intern Med;137: 631-639.

31. Wann LS, Curtis AB, January CT, Ellenbogen KA, Lowe JE, et al. (2011) 2011 ACCF/AHA/HRS focused update on the management of patients with atrial fibrillation (Updating the 2006 Guideline): a report of the American College of Cardiology Foundation/American Heart Association Task Force on Practice Guidelines. J Am Coll Cardiol;57: 223-242.

32. Witt BJ, Gami AS, Ballman KV, Brown RD Jr, Meverden RA, et al. (2007) The incidence of ischemic stroke in chronic heart failure: a meta-analysis. J Card Fail;13: 489-496.

33. Witt BJ, Brown RD Jr, Jacobsen SJ, Weston SA, Ballman KV, et al. (2006) Ischemic stroke after heart failure: a community-based study. Am Heart J;152: $102-109$.

34. Lip GY, Andreotti F, Fauchier L, Huber K, Hylek E, et al. (2011) Bleeding risk assessment and management in atrial fibrillation patients. Executive Summary of a Position Document from the European Heart Rhythm Association [EHRA], endorsed by the European Society of Cardiology [ESC] Working Group on Thrombosis. Thromb Haemost; 106: 997-1011.

35. European Heart Rhythm Association, European Association for CardioThoracic Surgery, Camm AJ, Kirchhof P, Lip GY, et al. (2010) Guidelines for the management of atrial fibrillation: the Task Force for the Management of Atrial Fibrillation of the European Society of Cardiology (ESC). Eur Heart J;31: 2369-2429. 